\title{
Handgrip Strength and Depression Among Older Chinese Inpatients: A Cross-Sectional Study
}

\author{
Xiao-Ming Zhang ${ }^{1, *}$ \\ Jing Jiao ${ }^{1, *}$ \\ Jing Cao' \\ $\mathrm{Na}$ Guo' \\ Chen Zhu' \\ Zhen $\mathrm{Li}^{\prime}$ \\ Xinjuan $\mathrm{Wu}^{\prime}$ \\ Tao $\mathrm{Xu}^{2}$
}

'Department of Nursing, Chinese Academy of Medical Sciences - Peking Union Medical College, Peking Union Medical College Hospital (Dongdan Campus), Beijing, 100730, People's Republic of China; ${ }^{2}$ Department of Epidemiology and Statistics, Institute of Basic Medical Sciences, Chinese Academy of Medical Sciences \& School of Basic Medicine, Peking Union Medical College, Beijing, 100005, People's Republic of China

*These authors contributed equally to this work
Correspondence: Xinjuan Wu; Tao Xu Tel +86 18612671300,

Fax +861069156114

Email wuxinjuan@sina.com;

xutaosd@I26.com
Objective: There have been studies exploring the association between handgrip and depression among community-dwelling adults; however, evidence for this association among hospitalized older adults is scarce. We aimed to use a larger-scale population from multiple centers to explore the association between low handgrip strength and depression.

Methods: This was a cross-sectional study that included hospitalized patients aged 65 years or older from different hospitals in China. Depression was assessed by a short-form Chinese Geriatric Depression Scale (GDS-15) with a cutoff value of 5 points or more. Dominant hand handgrip strength was assessed by a dynamometer. A generalized additive model and multivariable regression analysis were conducted.

Results: There were 9,368 participants, with an average age of $72.4(\mathrm{SD}=5.7)$ in the final analysis. The prevalence of low handgrip strength and depressive symptoms among older Chinese hospitalized patients was $50.3 \%$ and $16.3 \%$, respectively. There seemed to be a nonlinear relationship between handgrip strength and depression, and curve fitting and threshold analyses indicated that when handgrip strength was less than $35.6 \mathrm{~kg}$, the depression risk decreased significantly with increasing handgrip strength. However, this association between handgrip strength and depression was not significant when the handgrip strength was greater than $35.6 \mathrm{~kg}$. In addition, after adjusting for potential confounders, older patients with low handgrip strength had an increased risk of depression compared to those with normal handgrip strength $(\mathrm{OR}=1.46 ; 95 \% \mathrm{CI}=1.27-1.68)$. The subgroup analysis found the results were unchanged.

Conclusion: Our study indicates that low handgrip strength was associated with a higher risk of depression among older Chinese hospitalized patients, implying that early patient screening for handgrip strength and initiating effective intervention programs, such as resistance training and nutritional supplements, could be helpful for older patients.

Keywords: handgrip strength, depression, hospitalized, older adults

\section{Introduction}

Depression is a major public health issue worldwide. According to World Health Organization statistics, the number of depression cases was approximately 300 million, with an approximately $18 \%$ increase from 2005 to 2015 . $^{1}$ The number of cases among older adults will increase sharply as a result of aging societies worldwide. Previous studies have found that individuals with depression are at increased risk of adverse outcomes, lower quality of life, ${ }^{2}$ major complications, ${ }^{3}$ risk of suicide, ${ }^{4}$ and mortality, ${ }^{5}$ bringing about a large burden for society. Thus, identifying those at risk of depression and the management of depression are essential. 
Apart from all of the risk factors for depression, including genetics, serious illness, social factors, medication and lifestyle, ${ }^{6}$ muscle strength has become a research hotspot. ${ }^{7}$ Several studies have reported that muscle strength can secrete myokines, which are associated with the development of depression. ${ }^{8,9}$ Furthermore, muscle strength is a core component for maintaining function, which is closely associated with physical activity. ${ }^{10}$ Studies have confirmed that when patients participate in physical activity, it can reduce the risk of depression. ${ }^{11}$ Thus, there seems to be a relationship between muscle strength and depression. Handgrip strength is a simple and valid parameter that can reflect overall muscle strength and is widely used in the clinical setting. ${ }^{12}$ To date, the association between handgrip strength and depression has been widely explored. Many cross-sectional studies have found that adults with low handgrip strength were at increased risk of depression. ${ }^{13-16}$ Furthermore, three prospective cohort studies conducted in different countries have also reported similar results: high handgrip strength can reduce the risk of depression. ${ }^{17-19}$ Meanwhile, a recent meta-analysis showed that muscle strength has a positive impact on reducing depression, with a pooled OR of 0.85 (95\% CI: $0.80-0.89)^{7}$

However, most of the abovementioned studies were community-based or population-based. In fact, patients living in hospitals also suffer from depression, with a prevalence ranging from $5 \%$ to $60 \%$ and a median of $33 \%$ because of multiple comorbidities or long-term treatment, leading to more possibilities for developing adverse clinical outcomes. ${ }^{20}$ Therefore, the relationship between handgrip strength and depression is worth exploring. Furthermore, some geriatric syndromes, such as frailty, cognitive impairment and malnutrition, were reported to be associated with depression. ${ }^{21}$ A meta-analysis found that frailty increased the risk of depression, with a pooled OR of 1.90 (95\% CI: 1.55-2.32). ${ }^{22}$ However, these important confounding factors were seldom adjusted in previous studies, which might have overestimated the relationship between handgrip strength and depression. Accordingly, in a larger participant study from multiple centers, the object of our study was to explore the relationship between handgrip strength and depression among hospitalized patients when adjusting completed variables. Furthermore, we also reported this relationship when using low handgrip strength with the following cutoff values (male $<28 \mathrm{~kg}$ and female $<18 \mathrm{~kg}$ ) based on the Asian group of sarcopenias. ${ }^{23}$ We hypothesize that older inpatients with low handgrip strength were associated with a higher risk of depression among older Chinese hospitalized patients. We believe that our findings could contribute new evidence to this important issue.

\section{Materials and Methods Study Population and Sampling Method}

This study focused on hospitalized older patients from six tertiary class hospitals in China. The sampling method consisted of two stages, with simple random sampling representative of the Chinese population, which has been reported elsewhere. ${ }^{24}$ The inclusion criteria were as follows: age $\geq 65$ years old and hospitalized patients. We excluded participants who had endured long-term consistent unconsciousness and those who did not provide informed consent. Ethical approval was assessed and obtained by the review board of Peking Union Medical College Hospital with the number S-K540. In addition, all patients signed this written informed consent form when they agreed to participate in the study.

Our study was analyzed using baseline data and was conducted between October 2018 and February 2019. During this period, we initially enrolled 9,996 older participants from six hospitals. Participants completed a questionnaire that consisted of sociodemographic data, lifestyle factors, living ability and geriatric syndromes. We also collected disease history and anthropometric parameters, such as height, weight, and handgrip strength. For the purpose of our study, we excluded 682 patients who were unable to complete the handgrip strength and depression assessment.

\section{Measurement}

\section{Assessment of Handgrip Strength}

Trained nurses assisted participants in measuring handgrip strength. First, each nurse charged with collecting the data had attended a standard training program to ensure consistent quality. There were two ways for participants to measure handgrip strength using their dominant hand. Patients initially sat in a chair to keep their upper body straight with their elbow bending $90^{\circ}$ and then used their dominant hand to squeeze the dynamometer as hard as possible. Patients were asked to squeeze twice, recording the maximum number as the final data. Patients needing bedrest were asked to lie down on the bed, their elbow bending $30^{\circ}$, maintaining the lowest position supported by the bed, squeezing with the most strength possible, and 
recording the maximum number. According to the criteria of Asian Working Group for Sarcopenia, low handgrip strength is defined as $<28 \mathrm{~kg}$ for men and $<18 \mathrm{~kg}$ for women. $^{23}$

\section{Assessment of Depressive Symptoms}

A short-form Chinese Geriatric Depression Scale (GDS15) was adopted to assess the depressive symptoms of hospitalized older patients. ${ }^{25}$ This scale consists of 15 items, each item scoring 1 or 0 points, with the total score ranging from 0 to 15 . The cutoff value of the definition of depressive symptoms was 5 points or more. According to a previous study, the reliability and validity of this scale was good, with a Cronbach's $\alpha$ coefficient of 0.843 and retest reliability of $0.680 .^{26}$

\section{Definition of Covariates}

We used a questionnaire to collect potential confounders, including sociodemographic variables such as age, gender, ethnicity, education, marital status, and lifestyle factors, such as smoking status, alcohol consumption and BMI (weight/height ${ }^{2}$ ). Other covariates closely related to patient health, such as vision (normal versus dysfunctional), hearing (normal versus dysfunctional), sleep (normal versus dysfunctional), and urinary function (normal versus dysfunctional), were also assessed. We defined dysfunction of these covariates based on the conditions that influenced patient quality of life. Other important factors included a history of falls and whether patients had been diagnosed with cancer. To perform a statistical analysis, education was categorized into four levels: no formal schooling, primary school, middle school, and university; marital status was classified as married, divorced or widowed; smoking status was classified as nonsmoker, current smoker, or former smoker; and drinking was classified as nondrinker, current drinker, or former drinker.

We used different scales to assess geriatric syndromes, including cognitive impairment, frailty, and malnutrition. The Frail Scale included five items, covering fatigue, resistance, ambulation, illness, and loss of weight. ${ }^{27}$ The cutoff values of frailty, prefrailty and robustness were $\geq 3$, $1-2$, and 0 , respectively. Cognitive impairment was detected by the Mini-Cog scale consisting of two items: a three-item recall task and a clock drawing test, which was evaluated for memory and cognitive domains, respectively. ${ }^{28}$ The value of 2 was a cutoff for indicating cognitive impairment (0-2 points). Malnutrition was assessed using the Mini Nutritional Assessment - short form (MNA-SF) to screen nutritional status, with 0-7 points indicating malnourishment, 8-11 points indicating being at risk for malnutrition, and 12-14 points indicating normal nutritional status. ${ }^{29}$

\section{Statistical Analysis}

We used comprehensive methods to analyze the data. Data were classified as numerical and categorical and are displayed as the mean (standard deviation) and percentage, respectively. Student's $t$-test and the chi-squared test were used to compare the different variables between the two groups (depression versus nondepression and low handgrip strength versus normal handgrip strength). First, a generalized additive model (GAM) with smooth curve fitting ${ }^{30}$ was used to detect the association between handgrip strength and depression to identify whether there was a nonlinear relationship after adjusting for potential confounders. Trial and error methods, including selection of turning points using the recursive algorithm, were applied to determine the threshold. ${ }^{31}$ Then, we further used a twopiecewise regression model on both sides of the turning point to identify the threshold effects between handgrip strength and the risk of depression. Furthermore, we explored the association between low handgrip strength and depression in four models by adjusting for various factors. Model 1 was an unadjusted model; Model 2 adjusted for age, gender, education, marital status; Model3 adjusted for age, gender, education marital status, BMI, smoking, drinking, hearing, urinary function, vision, and sleeping; Model 4 was based on Model 3 plus frailty, cognitive impairment and nutritional status. All models used multivariable regression analysis with the results of odds ratios (ORs) and 95\% confidence intervals (CIs). In addition, we also performed a subgroup analysis to identify whether the association between low handgrip strength and depression was stable in different categorical variables, such as age (65-74 versus $\geq 75$ ), gender (female versus male), marital status (married versus divorced or widowed), education (four category levels), cancer patients (yes versus no), hearing (normal versus dysfunctional), sleep (normal versus dysfunctional), urinary function (normal versus dysfunctional), frailty (prefrailty, frailty, or nonfrail), cognitive impairment, and BMI $(<24$, 24-28 or $\geq 28$ ). We considered a two-tailed test with a $\mathrm{P}$ value $<0.05$ as having a significant difference. SAS version 9.4 (SAS Institute Inc., Cary, NC, USA) was used for all statistical analyses. 


\section{Results}

There were 9,368 participants in our final study based on our inclusion and exclusion criteria, with an average age of $72.4(\mathrm{SD}=5.7)$. Overall, the prevalence of depression was $16.3 \%$, and the average handgrip strength was $24.4 \mathrm{~kg}$ $(\mathrm{SD}=11.7)$. In this sample, the number of males $(58.0 \%)$ was higher than that of females $(42.0 \%)$, and most participants were married (88.7\%). A majority of patients were nonsmokers and nondrinkers. Most patients had attended or completed high school $(40.5 \%)$. For geriatric syndromes, the prevalence of frailty and cognitive impairment was about $20 \%$. Other detailed information is shown in Table 1. The comparison of variables was conducted in two groups (low handgrip strength versus normal strength), indicating significant differences in terms of variables. Patients with low handgrip strength tended to be older and female and suffered from frailty, cognitive impairment, and malnutrition (all $P<0.05$ ).

\section{Variable Comparison Between the Group with Depression and the Group without Depression}

The average handgrip strength in the group with depression was lower than that in the group without depression [19.8 $(\mathrm{SD}=10.2) \quad$ versus $25.3 \quad(\mathrm{SD}=11.8), \quad P<0.001]$. Overall, older hospitalized patients with depression were more likely to experience lower handgrip strength and a lower BMI value, with a significant difference, compared to those without depression. In addition, there were significant differences between those with depression and those without depression in terms of education, frailty, cognitive impairment, marital status, drinking, vision function, hearing function, sleep, urinary function, history of falls and nutritional status (all $P<0.05$ ). However, there was no significant difference between these two groups with regard to age, ethnicity or cancer (all $P>0.05$ ). As shown in Supplementary Table 1.

\section{Handgrip Strength and Depression}

We found that there seemed to be a nonlinear relationship between handgrip strength and depression, and the resulting curve displayed a two-stage change with a turning point. When the handgrip strength was less than the turning point, there was an inverse relationship between handgrip strength and depression. However, if the value was greater than the turning point, this inverse relationship was not significant (shown in Figure 1). Then, we used a two-piecewise linear regression model to examine the threshold effect of handgrip strength and depression according to the smoothing plot. As shown in Table 2, we further analyzed the threshold effect based on curve fitting, with the result indicating that the turning point was $35.6 \mathrm{~kg}$. When the handgrip strength value was less than $35.6 \mathrm{~kg}$, the OR value for the association between handgrip strength and depression was 0.95 (95\% CI: 0.95-0.96). However, this inverse relationship between handgrip strength and depression was not significant when the handgrip strength was greater than $35.6 \mathrm{~kg}$ ( $\mathrm{OR}=0.99$; 95\% CI: 0.97-1.01, $\mathrm{P}=0.287)$.

\section{Low Handgrip Strength and Depression}

The crude OR for the association between low handgrip strength and depression was 2.33 (95\% CI: 2.07-2.63) compared to those with normal handgrip strength. After adjusting for age, gender, education, and marital status, the association was marginally diminished ( $\mathrm{OR}=2.31 ; 2.04-2.62)$ (Model 2). In addition, this association also greatly decreased after adjusting for age, gender, education, marital status, BMI, smoking, drinking, hearing, urinary function, vision and sleep $\quad(\mathrm{OR}=2.02 ;$ 95\% CI: 1.78-2.30) (Model 3). Furthermore, we have added a fully adjusted model with the inclusion of frailty plus cognitive impairment and nutritional status into Model 4. The association greatly diminished, with an OR of 1.46 (95\% CI: 1.27-1.68), indicating that geriatric syndromes were confounding factors (Table 3 ). Furthermore, sensitivity analysis showed similar results when handgrip strength was a continuous variable or quartile of relative grip strength (Supplementary Table 2).

\section{Subgroup Analysis}

Figure 2 presents the results of the association between low handgrip strength and depression, stratified by a series of variables. Overall, we found that the effect of this association in each stratum was relatively unchanged, with no interaction effect in any of the variables $(P>0.05)$, indicating that this association was stable and reliable.

\section{Discussion}

Our study found a nonlinear association between handgrip strength and depression among older hospitalized patients. In addition, older inpatients aged 65 years old and up with low handgrip strength had a 1.46-fold higher risk of depression than patients with normal handgrip strength (low handgrip strength defined as $<28 \mathrm{~kg}$ for males and $<18 \mathrm{~kg}$ for females) after adjusting for completed 
Table I Baseline Characteristics (Overall and Low Handgrip Strength)

\begin{tabular}{|c|c|c|c|c|}
\hline Variables & $\begin{array}{l}\text { All Sample } \\
(n=9368)\end{array}$ & $\begin{array}{l}\text { Low Handgrip Strength } \\
(n=47 I I)\end{array}$ & $\begin{array}{l}\text { Normal Handgrip Strength } \\
(n=4657)\end{array}$ & $P$-value \\
\hline Age(years) (mean, SD) & $72.4 \pm 5.7$ & $73.6 \pm 6.1$ & $71.2 \pm 5.0$ & $<0.001$ \\
\hline BMI $\left(\mathrm{kg} / \mathrm{m}^{2}\right)$ (mean, SD) & $23.6 \pm 3.5$ & $23.0 \pm 3.6$ & $24 . I \pm 3.3$ & $<0.001$ \\
\hline Handgrip strength(kg) (mean, SD) & $24.4 \pm 11.7$ & $|7| \pm 6.2$. & $31.9 \pm 11.3$ & $<0.001$ \\
\hline Gender (n, \%) & & & & 0.032 \\
\hline Female & $3935(42.0 \%)$ & $2030(43.1 \%)$ & $1905(40.9 \%)$ & \\
\hline Male & $5433(58.0 \%)$ & $268 I(56.9 \%)$ & $2752(59.1 \%)$ & \\
\hline Education (n, \%) & & & & $<0.001$ \\
\hline No formal schooling & $1505(16.1 \%)$ & $963(20.4 \%)$ & $542(11.6 \%)$ & \\
\hline Primary & $2673(28.5 \%)$ & | 482(3|.5\%) & $1|9|(25.6 \%)$ & \\
\hline High & $3798(40.5 \%)$ & $1707(36.2 \%)$ & $209 \mid(44.9 \%)$ & \\
\hline University & $1390(14.9 \%)$ & $558(11.9 \%)$ & $832(17.9 \%)$ & \\
\hline Ethnicity (n, \%) & & & & $<0.001$ \\
\hline Han & $8804(94.0 \%)$ & $4366(92.7 \%)$ & $4438(95.3 \%)$ & \\
\hline Other & $564(6.0 \%)$ & $345(7.3 \%)$ & $219(4.7 \%)$ & \\
\hline Marital status (n, \%) & & & & $<0.001$ \\
\hline Wisdom or divorce & $1057(11.3 \%)$ & $622(13.2 \%)$ & $435(9.3 \%)$ & \\
\hline Marriage & $8300(88.7 \%)$ & $4083(86.8 \%)$ & $4217(90.7 \%)$ & \\
\hline Cancer (n, \%) & & & & 0.0007 \\
\hline Yes & $2489(26.6 \%)$ & $1179(25.0 \%)$ & $1310(28.1 \%)$ & \\
\hline No & $6879(73.4 \%)$ & $3532(75.0 \%)$ & $3347(71.9 \%)$ & \\
\hline Smoking (n, \%) & & & & $<0.001$ \\
\hline Nonsmoker & $6190(66.1 \%)$ & $3178(67.4 \%)$ & $30 \mid 2(64.7 \%)$ & \\
\hline Current smoker & $1029(11.0 \%)$ & $45 \mathrm{I}(9.6 \%)$ & $578(12.4 \%)$ & \\
\hline Former smoker & $2149(22.9 \%)$ & $1082(23.0 \%)$ & $1067(22.9 \%)$ & \\
\hline Drinking (n, \%) & & & & $<0.001$ \\
\hline Nondrinker & $7151(76.3 \%)$ & $3659(77.7 \%)$ & $3492(75.0 \%)$ & \\
\hline Current drinker & 1084(11.6\%) & $457(9.7 \%)$ & $627(13.5 \%)$ & \\
\hline Former drinker & $1133(12.1 \%)$ & $595(12.6 \%)$ & $538(I I .5 \%)$ & \\
\hline Fall history in last 12 months (n, \%) & & & & $<0.001$ \\
\hline No & $8060(86.0 \%)$ & $3916(83.1 \%)$ & $4 \mid 44(89.0 \%)$ & \\
\hline Yes & $1308(25.0 \%)$ & $795(16.9 \%)$ & $513(11.0 \%)$ & \\
\hline Vison function (n, \%) & & & & $<0.001$ \\
\hline Yes & 1948(20.8\%) & 1078(22.9\%) & $870(18.7 \%)$ & \\
\hline No & $7420(79.2 \%)$ & $3633(77.1 \%)$ & $3787(81.3 \%)$ & \\
\hline Hearing (n, \%) & & & & $<0.001$ \\
\hline Yes & $1688(18.0 \%)$ & $974(20.7 \%)$ & $7 \mid 4(15.3 \%)$ & \\
\hline No & $7688(82.0 \%)$ & $3737(79.3 \%)$ & $3943(84.7 \%)$ & \\
\hline Sleep function (n, \%) & & & & $<0.001$ \\
\hline Yes & $5342(57.0 \%)$ & $25 \mid 4(53.4 \%)$ & $2828(60.7 \%)$ & \\
\hline No & $4026(43.0 \%)$ & $2197(46.6 \%)$ & I829(39.3\%) & \\
\hline
\end{tabular}


Table I (Continued).

\begin{tabular}{|c|c|c|c|c|}
\hline Variables & $\begin{array}{l}\text { All Sample } \\
(n=9368)\end{array}$ & $\begin{array}{l}\text { Low Handgrip Strength } \\
(n=47 \mid I)\end{array}$ & $\begin{array}{l}\text { Normal Handgrip Strength } \\
(n=4657)\end{array}$ & $P$-value \\
\hline $\begin{array}{l}\text { Urinary function (n, \%) } \\
\text { Yes } \\
\text { no }\end{array}$ & $\begin{array}{l}8077(86.2 \%) \\
|29|(\mid 3.8 \%)\end{array}$ & $\begin{array}{l}3968(84.2 \%) \\
743(15.8 \%)\end{array}$ & $\begin{array}{l}4109(88.2 \%) \\
548(\mid 1.8 \%)\end{array}$ & $<0.001$ \\
\hline $\begin{array}{l}\text { Depression (n, \%) } \\
\text { Yes } \\
\text { No }\end{array}$ & $\begin{array}{l}1528(16.3 \%) \\
7840(83.7 \%)\end{array}$ & $\begin{array}{l}1036(22.0 \%) \\
3675(78.0 \%)\end{array}$ & $\begin{array}{l}492(10.6 \%) \\
4165(89.4 \%)\end{array}$ & \\
\hline $\begin{array}{l}\text { Frailty }(\mathrm{n}, \%) \\
\text { Yes } \\
\text { No }\end{array}$ & $\begin{array}{l}1609(17.2 \%) \\
7759(82.8 \%)\end{array}$ & $\begin{array}{l}\text { II75(24.9\%) } \\
3536(75.1 \%)\end{array}$ & $\begin{array}{l}434(9.3 \%) \\
4223(90.7 \%)\end{array}$ & $<0.001$ \\
\hline $\begin{array}{l}\text { Cognitive impairment }(\mathrm{n}, \%) \\
\text { Yes } \\
\text { No }\end{array}$ & $\begin{array}{l}1823(20.2 \%) \\
7203(79.8 \%)\end{array}$ & $\begin{array}{l}1199(26.8 \%) \\
3282(73.2 \%)\end{array}$ & $\begin{array}{l}624(13.7 \%) \\
3921(86.3 \%)\end{array}$ & $<0.001$ \\
\hline $\begin{array}{l}\text { MNASF (n, \%) } \\
\text { Normal nutritional } \\
\text { At risk of malnutrition } \\
\text { Malnourished }\end{array}$ & $\begin{array}{l}5116(54.6 \%) \\
3244(34.6 \%)) \\
1008(10.8 \%)\end{array}$ & $\begin{array}{l}2097(44.5 \%) \\
1884(40.0 \%) \\
730(15.5 \%)\end{array}$ & $\begin{array}{l}3019(64.8 \%) \\
1360(29.2 \%) \\
278(6.0 \%)\end{array}$ & $<0.001$ \\
\hline
\end{tabular}

Abbreviations: BMI, body mass index; MNA-SF, Mini Nutritional Assessment.

variables. To the best of our knowledge, this is the first patients, and we also reported a nonlinear relationship. large study to explore the association between handgrip Our study suggested that low handgrip strength is assostrength and depression among hospitalized Chinese ciated with a higher risk of depression, independent of

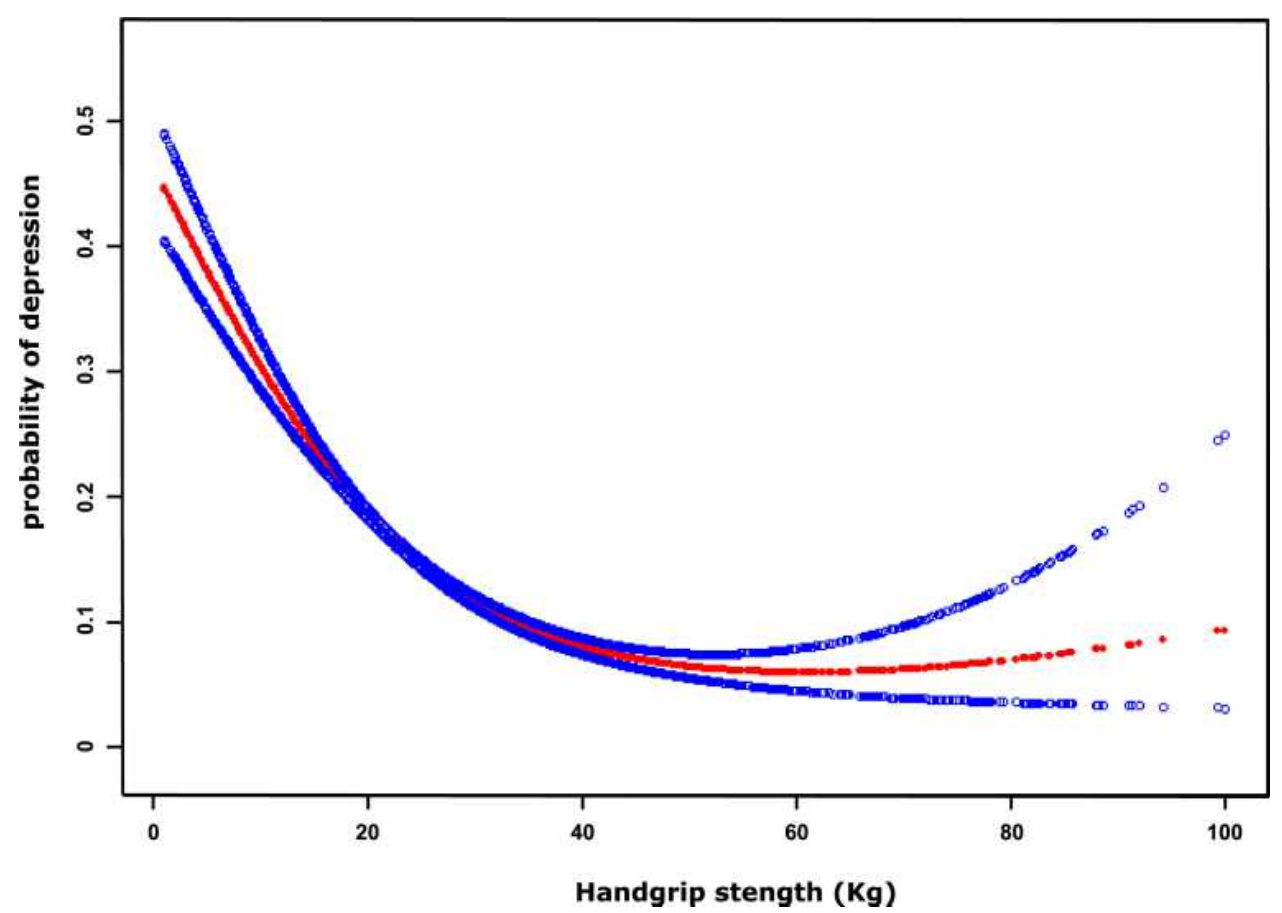

Figure I A nonlinear relationship between handgrip strength and probability of depression by a generalized additive model after adjusting for age, sex, BMI, frailty, and cognitive impairment. The red dotted lines represent the estimated risk of depression, and the blue dotted lines indicate the $95 \% \mathrm{Cl}$ of the spline plots. 
Table 2 Threshold Effect Analysis of Handgrip Strength on Depression Using a Two-Piecewise Regression Model

\begin{tabular}{|l|l|l|}
\hline Outcome & $\begin{array}{l}\text { Depression Adjusted } \\
\text { OR (95\% Cl) }\end{array}$ & P-value \\
\hline $\begin{array}{l}\text { Fitting model by two- } \\
\text { piecewise linear regression }\end{array}$ & $35.6 \mathrm{~kg}$ & \\
\hline $\begin{array}{l}\text { Turning point of handgrip } \\
\text { strength } \\
\text { STurning point }\end{array}$ & $0.95(0.95,0.96)$ & $<0.001$ \\
>Turning point & $0.99(0.97,1.01)$ & 0.287 \\
\hline
\end{tabular}

Note: Adjusted for age, gender, BMI, frailty, and cognitive impairment. was considered according to ethnicity and lifestyle, and although this indicator adopted different cutoff points, it generated similar results, making the association between handgrip strength and depression conclusive. Furthermore, there have been several prospective cohort studies from different countries, such as China, Ireland, Italy, and Japan, exploring the association between handgrip strength and depression. ${ }^{17-19,33}$ Among these four studies, only one study, which covered participants from 12 European countries, reported no significant association among baseline low handgrip strength and depression at the four-year follow-

Table 3 Multivariable Regression for the Association Between Low Handgrip Strength and Depression by Adjusting Different Variables

\begin{tabular}{|l|l|l|l|l|}
\hline & Model I & Model 2 & Model 3 & Model 4 \\
\hline $\begin{array}{l}\text { Low handgrip strength } \\
\text { No }\end{array}$ & & & & \\
Yes & Reference & Reference & Reference & Reference \\
\hline
\end{tabular}

Notes: Model I, unadjusted; Model 2, age gender education, Marital status; Model 3, Age gender education, marital status, BMI, smoking, drinking, hearing, Urinary function, vision, sleeping; Model 4, Age gender education, marital status, BMI, smoking, drinking, hearing, Urinary function, vision, sleeping, frailty, cognitive impairment, and malnutrition.

sociodemographic, lifestyle, geriatric syndromes, and other confounding factors.

The impact of handgrip strength on depression has been examined in several studies, mainly focusing on community dwelling adults from various countries. ${ }^{13-16}$ In a crosssectional study with a total of 24,109 Chinese adults, the authors reported that male participants with the highest grip strength were not at a reduced risk of depression $(\mathrm{OR}=0.91$; 95\% CI: 0.78-1.07) compared to male participants with the lowest grip strength; however, this association of a reduced risk of depression was found in females. ${ }^{14}$ Another study of adults aged 50 years and older reported that both females and males had a significantly reduced risk of depression in all grip strength quartiles, 2,3 , and 4, compared to those in the lowest quartiles. ${ }^{15}$ In addition, a study with a larger sample of individuals aged $>50$ years from different countries found that participants with weak handgrip strength had a higher risk of depression $(\mathrm{OR}=1.45,95 \% \mathrm{CI}: 1.12-1.88),{ }^{13}$ which is in line with our study. Moreover, the authors used the definition of European Working Group on Sarcopenia in Older People, indicating weak handgrip strength as $<30 \mathrm{~kg}$ for men and $<20 \mathrm{~kg}$ for women. ${ }^{32}$ Our study used the Asian Working Group for Sarcopenia definition ( $<28 \mathrm{~kg}$ for men and $<18 \mathrm{~kg}$ for women). The definition of low handgrip strength in different geographic regions (EWGOSP versus AWGS) up. ${ }^{19}$ To date, a large number of new studies have suggested that higher handgrip strength is associated with reduced risk of depression. Recently, a new meta-analysis reported an inverse and significant relationship between muscle strength and depression, with an OR of 0.85 (95\% CI: $0.80-0.89){ }^{7}$ which provides evidence supporting the impact of handgrip on depression. However, a majority of these studies only focused on community-dwelling patients, and few studies concentrated on hospitalized participants. In fact, hospitalized patients usually suffer from worse disease conditions and experience multiple geriatric syndromes. Our study indicated that the hospitalization of older patients with low handgrip strength was associated with a higher risk of depression, which serves as a reminder to medical personnel to pay more attention to hospitalized older patients. Early screening of handgrip strength and encouraging patients to undertake effective physical exercise is a golden opportunity for reducing the likelihood of depression.

To the best of our knowledge, our study is the first to find a nonlinear relationship between handgrip and depression. After adjusting for age, gender, BMI, frailty, and cognitive impairment, the maximum benefit cutoff value for improving handgrip strength to reduce the risk of depression among hospitalized patients was $35.6 \mathrm{~kg}$. Furthermore, with the increase in handgrip strength at a threshold value 


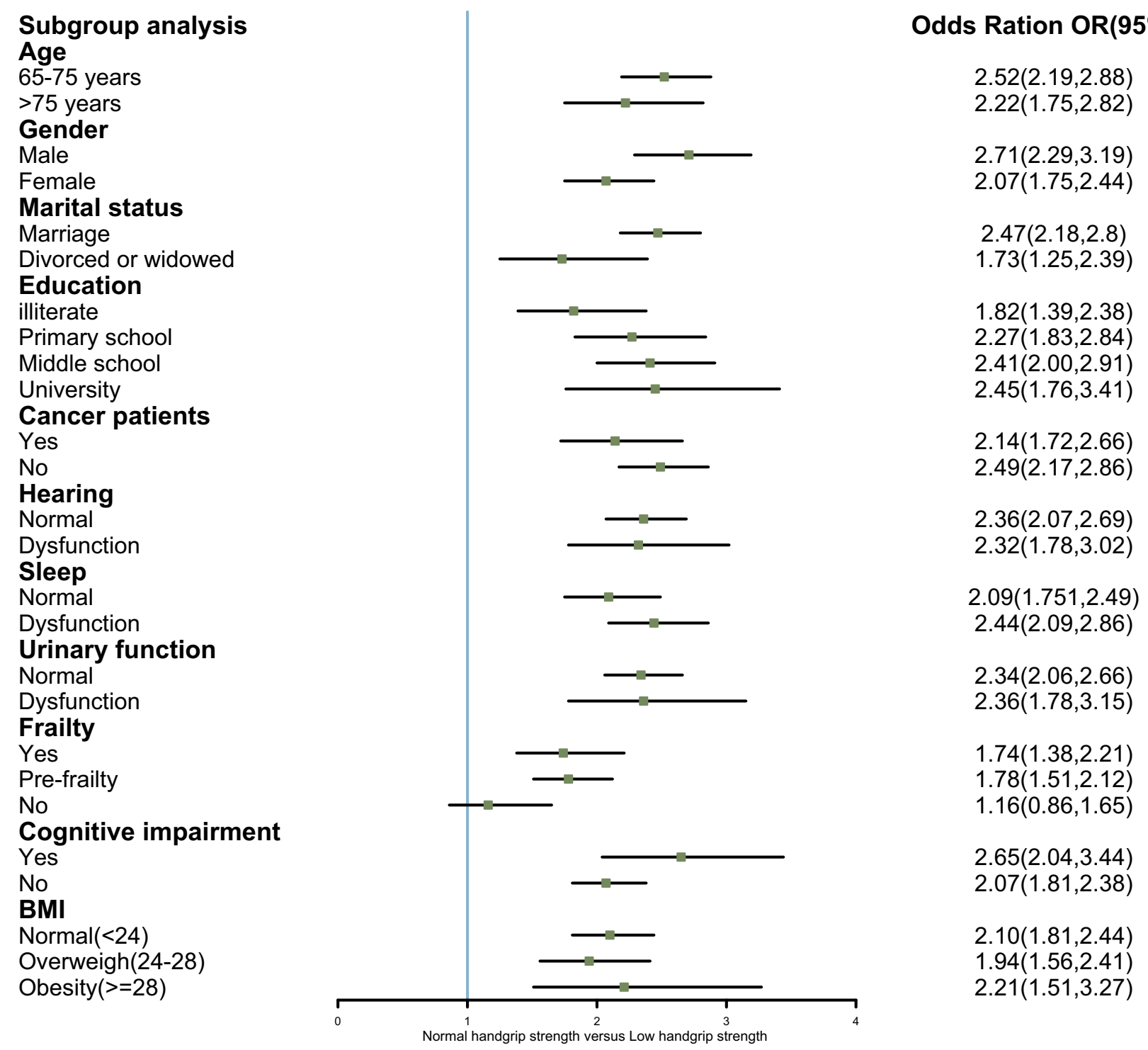

Figure 2 Subgroup analysis of the association between low handgrip strength and depression.

$(>35.6 \mathrm{~kg})$, the effect of reducing the rate of depression was not significant and might be steadily sustained at a lower level, which was consistent with the physiopsychological phenomenon. This is because apart from lower handgrip strength, other important factors that influence depression, such as genetics, multimorbidity, social factors, and medication, could still exist. Our study suggests that improving handgrip strength is beneficial for reducing the likelihood of depression, but there is a saturation threshold for handgrip strength, which can serve as an appropriate handgrip strength target for individuals. Because this was a cross-sectional study design, a future large-sample prospective cohort and randomized controlled trial should be conducted to verify this important issue.
A previous study found that abdominal obesity (waist circumference) exerts a modification effect on both handgrip strength and depression, reminding us that obesity combined with low handgrip strength exerts a cumulative effect on the risk of depression. ${ }^{34}$ In addition, other studies have reported that depression combined with obesity among older adults can lead to lower handgrip strength. ${ }^{35}$ Our study did not find any differences in any BMI stratum. There is a new concept of sarcopenic obesity, with the characteristics of declining muscle strength and increased obesity resulting in a higher risk of adverse outcomes compared to normal conditions. More studies are needed in the future to explore the association between handgrip strength, BMI, and depression. 
Our study has several clinical implications. First, given that our results indicate the importance of improving handgrip strength, screening for handgrip strength may have multiple benefits. It is assumed that depression is harmful to patient health. Prevention and management of depression is essential. Given that depression is a reversible symptom, reducing the risk factors is a priority task. Low handgrip strength is a potential risk factor for depression; thus, effective physical activity could be beneficial. Resistance training exercises and nutritional programs help people enhance handgrip strength. A meta-analysis indicated that task-specific training modes may have the potential effect of improving handgrip strength. ${ }^{36}$ In addition, a study revealed that the relationship between handgrip strength might be bidirectional. ${ }^{37}$ When patients suffer from depressive symptoms, they are unlikely to take part in physical activity, which might influence their sleep quality, possibly leading to a decline in muscle strength. ${ }^{35}$ Studies have shown that physical activity can alleviate depression. Therefore, exercise training programs not only improve handgrip strength but also have a potential benefit for depression, which might exert a cumulative effect on both handgrip strength and depression.

The mechanism for the association between handgrip strength and depression is complicated and has been described in previous studies. ${ }^{7,13}$ First, low handgrip strength is an indicator of sarcopenia, which is strongly related to adverse outcomes, such as major complications, extended hospital stays, and lower quality ${ }^{38}$ of life, thus increasing the likelihood of depression when patients experience these adverse clinical outcomes. A meta-analysis reported that sarcopenia increases the risk of depression (pooled $\mathrm{OR}=1.821,95 \% \mathrm{CI}$ : $1.16-2.85) .{ }^{39}$ Second, another possible reason is that muscles can excrete myokines, circulating from blood to the brain to regulate the cerebrum's function, which helps reduce depression $^{40}$ rates. Some studies have found myokines, such as brain-derived neurotrophic factor and irisin, through specific signaling pathways to mediate depression symptoms. ${ }^{9,41}$ Finally, low handgrip strength influences depression through psychosocial mechanisms. Older adults with low handgrip strength usually experience a decline in physical ability and function, which limits their opportunities to participate in outdoor activities, decreasing possible social contact with other people. $^{42,43}$ They might be more likely to develop depression through a vicious circle. In summary, the mechanism for these associations is complex, and we were unable to draw a conclusion.

Our study has several strengths and drawbacks. First, this is a large multiple center study from China with minimal selective bias based on random sampling. To the best of our knowledge, we found a nonlinear relationship between handgrip strength and depression among older Chinese hospitalized patients, which could add new evidence to this field. Second, previous studies found that geriatric syndromes, such as frailty, cognitive impairment and malnutrition, are associated with depression. ${ }^{21,22}$ Therefore, we adjusted for confounding factors, including age, sex, education, marital status, BMI, smoking, drinking, hearing, urinary function, vision, sleeping, frailty, cognitive impairment, and malnutrition, which helped to calculate the independent effect of low handgrip strength on depression. In comparison, previous studies did not adjust for these geriatric syndromes (potential confounding factors). However, several limitations must also be noted. First, this is a cross-sectional study and cannot assess causality. Second, we did not collect other confounding factors, such as a family history of mental illness, which could have overestimated the effect of handgrip strength on depression. Third, this study only investigated a Chinese population, which may limit generalization to other counties. Fourth, the assessment of depression was based on self-reported methods (subjective), which might have led to errors in diagnosing depression. A prospective large-scale cohort study based on patients in hospitals is urgently required to explore this issue further.

\section{Conclusion}

Our study indicates that low handgrip strength is associated with an increased risk of depression among hospitalized populations, which serves as a reminder to medical personnel to conduct early screening for handgrip strength and effective interventions, such as resistance training or nutritional programs, to reduce depressive symptoms in older adults, which may help alleviate the health care system burden.

\section{Data Sharing Statement}

The data used in this current study are available from the corresponding author Xinjuan Wu upon reasonable request (wuxinjuan@sina.com).

\section{Ethics and Consent Statement}

The study was approved by the Ethics Committee of Peking Union Medical College Hospital number S-K540). In addition, all patients signed this written informed consent form when they agreed to participate in the study. The authors confirm that this study was conducted in accordance with the Declaration of Helsinki. 


\section{Acknowledgments}

We sincerely thank all of the participants in our program and thank the Nursing Department of Peking Union Medical College for its support.

Co-first authors: Xiao-Ming Zhang and Jing Jiao. Cocorresponding authors: Xinjuan $\mathrm{Wu}$ and Tao Xu.

\section{Funding}

This work was supported by Peking Union Medical College, which funded this study, with grant number 2018 PT33001.

\section{Disclosure}

All authors declare no conflicts of interest for this work.

\section{References}

1. Perini G, Cotta Ramusino M, Sinforiani E, Bernini S, Petrachi R, Costa A. Cognitive impairment in depression: recent advances and novel treatments. Neuropsychiatr Dis Treat. 2019;15:1249-1258. doi:10.2147/NDT.S199746

2. Chang HK, Gil CR, Kim HJ, Bea HJ. Factors affecting quality of life among the elderly in long-term care hospitals. J Nurs Res. 2020;29. doi:10.1097/jnr.0000000000000413

3. Ghoneim MM, O'Hara MW. Depression and postoperative complications: an overview. BMC Surg. 2016;16:5. doi:10.1186/s12893-0160120-y

4. Ferrari AJ, Charlson FJ, Norman RE, et al. Burden of depressive disorders by country, sex, age, and year: findings from the global burden of disease study 2010. PLoS Med. 2013;10(11):e1001547. doi:10.1371/journal.pmed.1001547

5. Walker ER, McGee RE, Druss BG. Mortality in mental disorders and global disease burden implications: a systematic review and meta-analysis. JAMA Psychiatry. 2015;72(4):334-341. doi:10.1001/ jamapsychiatry.2014.2502

6. Meng X, Brunet A, Turecki G, Liu A, D'Arcy C, Caron J. Risk factor modifications and depression incidence: a 4-year longitudinal Canadian cohort of the Montreal Catchment Area Study. BMJ Open. 2017;7(6):e015156.

7. Marques A, Gomez-Baya D, Peralta M, et al. The effect of muscular strength on depression symptoms in adults: a systematic review and meta-analysis. Int J Environ Res Public Health. 2020;17(16).

8. Han DS, Hsiao MY, Wang TG, Chen SY, Yang WS. Relation between serum myokines and phase II cardiac rehabilitation. Medicine. 2017;96(16):e6579. doi:10.1097/MD.0000000000006579

9. Tu WJ, Qiu HC, Liu Q, Li X, Zhao JZ, Zeng X. Decreased level of irisin, a skeletal muscle cell-derived myokine, is associated with post-stroke depression in the ischemic stroke population. $J$ Neuroinflammation. 2018;15(1):133. doi:10.1186/s12974-018-1177-6

10. Brill PA, Macera CA, Davis DR, Blair SN, Gordon N. Muscular strength and physical function. Med Sci Sports Exerc. 2000;32 (2):412-416. doi:10.1097/00005768-200002000-00023

11. Choi KW, Chen C-Y, Stein MB, et al. Assessment of bidirectional relationships between physical activity and depression among adults: a 2-sample mendelian randomization study. JAMA Psychiatry. 2019;76(4):399-408. doi:10.1001/jamapsychiatry.2018.4175

12. Samuel D, Wilson K, Martin HJ, Allen R, Sayer AA, Stokes M. Ageassociated changes in hand grip and quadriceps muscle strength ratios in healthy adults. Aging Clin Exp Res. 2012;24(3):245-250. doi:10.1007/BF03325252
13. Ashdown-Franks G, Stubbs B, Koyanagi A, et al. Handgrip strength and depression among 34,129 adults aged 50 years and older in six low- and middle-income countries. $J$ Affect Disord. 2019;243:448-454. doi:10.1016/j.jad.2018.09.036

14. Gu Y, Li X, Zhang Q, et al. Grip strength and depressive symptoms in a large-scale adult population: the TCLSIH cohort study. J Affect Disord. 2020;279:222-228. doi:10.1016/j.jad.2020.08.023

15. Marques A, Gaspar de Matos M, Henriques-Neto D, et al. Grip strength and depression symptoms among middle-age and older adults. Mayo Clin Proc. 2020;95(10):2134-2143. doi:10.1016/j. mayocp.2020.02.035

16. Chen L, Sheng Y, Qi H, Tang T, Yu J, Lv S. Correlation of sarcopenia and depressive mood in older community dwellers: a cross-sectional observational study in China. BMJ Open. 2020;10(9):e038089. doi:10.1136/bmjopen-2020-038089

17. Zhao Z, Ji C, Liu Y, Gao S, Xia Y. Higher handgrip strength predicts a lower risk of depressive symptoms in rural Chinese populations. J Affect Disord. 2020;269:12-17. doi:10.1016/j.jad.2020.03.028

18. Carvalho AF, Maes M, Solmi M, et al. Is dynapenia associated with the onset and persistence of depressive and anxiety symptoms among older adults? Findings from the Irish longitudinal study on ageing. Aging Ment Health. 2021;23:468-475.

19. Bertoni M, Maggi S, Manzato E, Veronese N, Weber G. Depressive symptoms and muscle weakness: a two-way relation? Exp Gerontol. 2018;108:87-91. doi:10.1016/j.procir.2018.03.218

20. IsHak WW, Collison K, Danovitch I, et al. Screening for depression in hospitalized medical patients. J Hosp Med. 2017;12(2):118-125. doi:10.12788/jhm.2693

21. Vafaei Z, Mokhtari H, Sadooghi Z, Meamar R, Chitsaz A, Moeini M. Malnutrition is associated with depression in rural elderly population. J Res Med Sci. 2013;18(Suppl 1):S15-S19.

22. Soysal P, Veronese N, Thompson T, et al. Relationship between depression and frailty in older adults: a systematic review and meta-analysis. Ageing Res Rev. 2017;36:78-87.

23. Chen LK, Woo J, Assantachai P, et al. Asian working group for sarcopenia: 2019 consensus update on sarcopenia diagnosis and treatment. J Am Med Dir Assoc. 2020;21(3):300-307.e302. doi:10.1016/j.jamda.2019.12.012

24. Jiao J, Wang Y, Zhu C, et al. Prevalence and associated factors for frailty among elder patients in China: a multicentre cross-sectional study. BMC Geriatr. 2020;20(1):100. doi:10.1186/s12877-020-1496-1

25. Chau J, Martin CR, Thompson DR, Chang AM, Woo J. Factor structure of the Chinese version of the geriatric depression scale. Psychol Health Med. 2006;11(1):48-59. doi:10.1080/ 13548500500093688

26. Xie Z, Lv X, Hu Y, et al. Development and validation of the geriatric depression inventory in Chinese culture. Int Psychogeriatr. 2015;27 (9):1505-1511. doi:10.1017/S1041610215000162

27. Nicholson C, Morrow EM, Hicks A, Fitzpatrick J. Supportive care for older people with frailty in hospital: an integrative review. Int J Nurs Stud. 2017;66:60-71. doi:10.1016/j.ijnurstu.2016.11.015

28. Li X, Dai J, Zhao S, Liu W, Li H. Comparison of the value of Mini-Cog and MMSE screening in the rapid identification of Chinese outpatients with mild cognitive impairment. Medicine. 2018;97(22):e10966. doi:10.1097/MD.0000000000010966

29. Kaiser MJ, Bauer JM, Ramsch C, et al. Validation of the Mini Nutritional Assessment short-form (MNA-SF): a practical tool for identification of nutritional status. J Nutr Health Aging. 2009;13 (9):782-788. doi:10.1007/s12603-009-0214-7

30. Roberto Maass-Moreno R. Fitting Models to biological data using linear and nonlinear regression: a practical guide to curve fitting. By Harvey Motulsky and Arthur Christopoulos. Q Rev Biol. 2005;80 (1): 101.

31. Shi P, Jing H, Xi S. Urinary metal/metalloid levels in relation to hypertension among occupationally exposed workers. Chemosphere. 2019;234:640-647. doi:10.1016/j.chemosphere.2019.06.099 
32. Cruz-Jentoft AJ, Bahat G, Bauer J, et al. Sarcopenia: revised European consensus on definition and diagnosis. Age Ageing 2019;48(1):16-31. doi:10.1093/ageing/afy169

33. Fukumori N, Yamamoto Y, Takegami M, et al. Association between hand-grip strength and depressive symptoms: Locomotive Syndrome and Health Outcomes in Aizu Cohort Study (LOHAS). Age Ageing. 2015;44(4):592-598. doi:10.1093/ageing/afv013

34. Kokkeler KJE, van den Berg KS, Comijs HC, Oude Voshaar RC, Marijnissen RM. Sarcopenic obesity predicts nonremission of late-life depression. Int $J$ Geriatr Psychiatry. 2019;34(8):1226-1234. doi:10.1002/gps.5121

35. Smith L, White S, Stubbs B, et al. Depressive symptoms, handgrip strength, and weight status in US older adults. J Affect Disord. 2018;238:305-310. doi:10.1016/j.jad.2018.06.016

36. Labott BK, Bucht H, Morat M, Morat T, Donath L. Effects of exercise training on handgrip strength in older adults: a meta-analytical review. Gerontology. 2019;65(6):686-698. doi:10.1159/000501203

37. Lee K. Relative handgrip strength in relation to depressive mood and suicidal ideation in Koreans using the 2015 KNHANES data. J Musculoskelet Neuronal Interact. 2018;18(3):333-338.
38. Xia L, Zhao R, Wan Q, et al. Sarcopenia and adverse health-related outcomes: an umbrella review of meta-analyses of observational studies. Cancer Med. 2020;9(21):7964-7978. doi:10.1002/cam4.3428

39. Chang KV, Hsu TH, Wu WT, Huang KC, Han DS. Is sarcopenia associated with depression? A systematic review and meta-analysis of observational studies. Age Ageing. 2017;46(5):738-746. doi:10.1093/ageing/afx094

40. Schnyder S, Handschin C. Skeletal muscle as an endocrine organ: PGC-1 $\alpha$, myokines and exercise. Bone. 2015;80:115-125. doi:10.1016/j.bone.2015.02.008

41. Colucci-D’Amato L, Speranza L, Volpicelli F. Neurotrophic factor BDNF, physiological functions and therapeutic potential in depression, neurodegeneration and brain cancer. Int J Mol Sci. 2020;21 (20):7777. doi:10.3390/ijms21207777

42. Kozakai R. Grip strength and healthy aging. J Phys Fitness Sports Med. 2017;6(3):145-149. doi:10.7600/jpfsm.6.145

43. Almquist YB, Landstedt E, Hammarström A. Associations between social support and depressive symptoms: social causation or social selection-or both? Eur J Public Health. 2017;27(1):84-89. doi:10.1093/eurpub/ckw120

\section{Publish your work in this journal}

Neuropsychiatric Disease and Treatment is an international, peerreviewed journal of clinical therapeutics and pharmacology focusing on concise rapid reporting of clinical or pre-clinical studies on a range of neuropsychiatric and neurological disorders. This journal is indexed on PubMed Central, the 'PsycINFO' database and CAS, and is the official journal of The International Neuropsychiatric Association (INA). The manuscript management system is completely online and includes a very quick and fair peer-review system, which is all easy to use. Visit http://www.dovepress.com/testimonials.php to read real quotes from published authors. 